\title{
Mathematical Modeling of Heat-Transfer For a Moving Sheet in a Moving Fluid
}

\author{
H. M. Habib ${ }^{1,2}$ and E. R. El-Zahar ${ }^{1,2 \dagger}$ \\ ${ }^{1}$ Department of Mathematics, Faculty of Science and Humanity Studies, Salman Bin AbdulAziz University, P. O. Box \\ 83, Alkharj11942, KSA. \\ ${ }^{2}$ Department of Basic Engineering Science, College of Engineering, Menoufia University, Shebin El-Kom, Egypt.
}

†Corresponding Author Email: essam_zahar2006@yahoo.com

(Received November 28, 2011; accepted April 16, 2012)

\begin{abstract}
A mathematical model was developed for determining the heat transfer between a moving sheet that passes through a moving fluid environment to simulate the fabrication process of sheet and fiber-like materials. Similarity transformations were introduced to reduce the governing equations to two nonlinear ordinary differential equations. For high values Prandtl number, the energy equation became much stiffer or singularly perturbed and the standard numerical methods failed to handle it. An innovative procedure combining shooting and singular perturbation technique was developed. The results show that the heat transfer depends on the relative velocity between the moving fluid and the moving sheet to a certain value after that value the relative velocity has no effect. If blowing effect is found the thermal layer becomes thinner and temperature profiles are backed together.
\end{abstract}

Keywords: Heat transfer, Boundary layer, Singular perturbation

\section{NOMENCLATURE}

$\begin{array}{ll}C & \text { specific heat } \\ f & \text { fluid } \\ K & \text { thermal conductivity } \\ P & \text { pressure } \\ P r & \text { Prandtl Number } \\ r e l & \text { relative } \\ S & \text { surface } \\ T & \text { temperature } \\ U & \text { velocity in the stream-wise direction } \\ X & \text { coordinate in flow direction }\end{array}$

\section{INTRODUCTION}

In many manufacturing processes, such as hot rolling, drawing, extrusion, and continuous casting, heat transfer occurs in a continuously moving material. In the fabrication of sheet and fiber like materials, the material customarily is in motion during the manufacturing process. Typical materials include polymer sheets, paper, linoleum, roofing shingles, and fine-fiber mats. The processing may involve heat transfer between the material and an adjacent fluid, which may also be in motion. In order to complete the finishing operation, a fluid flows over the sheet either to heat or cool it before rollup. In virtually all such processing operations, the sheet moves parallel to its own plane. The moving sheet may induce motion in the neighboring fluid or, alternatively, the fluid may have an independent forced-convection motion that is parallel to that of the sheet. Representative applications involving a moving sheet and an independently moving fluid are illustrated in Figure 1. In Figure 1, an unfinished or partially finished material is unrolled and becomes a moving sheet.

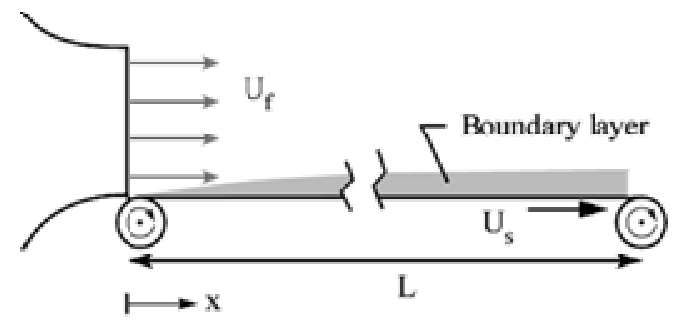

Fig. 1. Processing station consisting of a moving sheet 
situated in a parallel gas flow

Heat transfer between the sheet and the adjacent fluid is usually initiated at the first contact between the media. If the fluid temperature is lower than that of the sheet, the sheet temperature decreases in the streamwise direction. Alternatively, if the fluid temperature is greater than the sheet temperature, there is a streamwise increase of the latter. The design of a thermal processing station for moving sheets requires knowledge of heat transfer rates and corresponding sheet temperature variations. In practice, it is highly likely that the thermal interaction between the moving sheet and the moving fluid would give rise to a streamwise variation of the sheet temperature.

The literature on fluid flow and heat transfer for the moving sheet problem can be conveniently classified into two groups: (1) heat transfer in the fluid corresponding to a prescribed, artificial temperature boundary condition at the fluid-sheet interface without thermal participation of the sheet, and (2) coupled heat transfer in the fluid and in the moving sheet for the special case of a moving sheet in an otherwise quiescent fluid. With regard to group 1, (Sakiadis 1961, Tsou et al. 1967, Abdelhafez 1985, Chen 1999), the thermal boundary condition, which was most often employed, is the isothermal sheet. Chen (2000) assumed the difference between the surface temperature and the fluid temperature to vary as a power of the streamwise coordinate. This prescribed temperature was chosen in order to obtain a similarity solution for the temperature field.

The papers of group 2 (Chida and Katto 1976, Karwe and Jaluria 1986, Char et al. 1990, Karwe and Jaluria 1991, 1992, Mendez and Trevino 2002) represent, for most part, numerical solutions of the coupled energy equations for the moving sheet and induced boundary layer flow in the otherwise quiescent fluid. Mendez and Trevino (2002) employed an integral method while Karwe and Jaluria (1991) used a simplified model in which a known heat transfer coefficient was imposed on the moving sheet. Although the papers of group 2 dealt with the conjugate problem in some form, all except Karwe and Jaluria (1991) and Mendez and Trevino (2002) required the solution of differential equations. Abraham and Sparrow (2005) developed universal solutions for the stream-wise variation of the temperature of a moving sheet in the presence of a moving fluid. In this paper a special treatment for the energy equation was used for high Prandtl numbers, where the equation became much stiffer or singularly perturbed and the standard numerical methods failed to handle it.

\section{GOVERNING EQUATIONS}

Consider steady, laminar flow of viscous, incompressible fluid, over an isothermal surface, as illustrated in Fig. 1. Making the standard boundary layer assumptions, conservation of mass, momentum and energy flow are:

Continuity:

$\frac{\partial u}{\partial x}+\frac{\partial v}{\partial y}=0$
Momentum:

$u \frac{\partial u}{\partial x}+v \frac{\partial u}{\partial y}=-\frac{1}{\rho} \frac{\partial p}{\partial x}+\nu \frac{\partial^{2} u}{\partial y^{2}}$

Energy:

$u \frac{\partial T}{\partial x}+v \frac{\partial T}{\partial y}=\alpha \frac{\partial^{2} T}{\partial y^{2}}$.

Subject to the boundary conditions:

at $y=0 \quad u=U_{s}, \quad v=0$, and $\quad T=T_{s}$

at $y \rightarrow \infty \quad u=U_{f}$ and $\quad T=T_{f}$

In these equations, $u$ and $v$ are the velocity components that respectively correspond to $x$ and $y$, with $\mathrm{p}$ the pressure, and $\rho$ and $\nu$, respectively, the density and the viscosity of the fluid. Although both the free-stream flow and the bounding surface move, the stream-wise pressure gradient $\partial p / \partial x$ is essentially zero, as it is for the two limiting cases of a moving fluid in the presence of a stationary surface and a moving surface passing through a stationary fluid. These equations may be transformed from the realm of partial differential equations to that of ordinary differential equations by using the similarity transformation from the $x-y$ plane to the $\eta$ plane.

Introducing the stream function $\psi$ as

$$
u=\frac{\partial \psi}{\partial y}, v=-\frac{\partial \psi}{\partial x}
$$

eliminates the continuity equation and transforms the momentum and energy equations to

$$
\begin{aligned}
& \frac{\partial \psi}{\partial y} \frac{\partial^{2} \psi}{\partial x \partial y}-\frac{\partial \psi}{\partial x} \frac{\partial^{2} \psi}{\partial y^{2}}=v \frac{\partial^{3} \psi}{\partial y^{3}} \\
& \frac{\partial \psi}{\partial y} \frac{\partial T}{\partial x}-\frac{\partial \psi}{\partial x} \frac{\partial T}{\partial y}=\alpha \frac{\partial^{2} T}{\partial y^{2}}
\end{aligned}
$$

Making the standard coordinate transformation

$$
\eta=\left(\frac{u_{r e l}}{\nu x}\right)^{\frac{1}{2}} y
$$

and introducing new non-dimensional dependent variables

$$
f=\frac{\psi}{\left(v u_{r e l} x\right)^{\frac{1}{2}}}, \theta=\frac{T(x, y)-T_{f}}{T_{s}-T_{f}}
$$

reduces momentum and energy equations to

$$
\frac{d^{3} f}{d \eta^{3}}+\frac{1}{2} f \frac{d^{2} f}{d \eta^{2}}=0
$$




$$
\frac{1}{p_{r}} \frac{d^{2} \theta}{d \eta^{2}}+\frac{1}{2} f \frac{d \theta}{d \eta}-\frac{d f}{d \eta} \theta=0
$$

In situations like transpiration cooling the transverse component of the velocity is not zero and can vary as a power of the streamwise coordinate in order to obtain similarity for $\mathrm{f}$.

The boundary conditions are:

$$
\begin{aligned}
& \text { at } \eta=0 \quad f=0, \quad f^{\prime}=\frac{U_{s}}{U_{r e l}}, \text { and } \quad \theta=1 \\
& \text { at } \eta \rightarrow \infty \quad, f^{\prime}=\frac{U_{f}}{U_{r e l}} \text {, and } \quad \theta=0
\end{aligned}
$$

where $U_{r e l}=\left|U_{f}-U_{s}\right|$.

Define $\alpha=\frac{U_{f}}{U_{r e l}}$, then

$$
\left.\begin{array}{l}
f^{\prime}(0)=\frac{U_{s}}{U_{r e l}}=\frac{1}{\left|1-U_{f} / U_{s}\right|}=\frac{1}{|1-\alpha|}, \\
f^{\prime}(\infty)=\frac{U_{f}}{U_{r e l}}=\frac{U_{f} / U_{s}}{\left|1-U_{f} / U_{s}\right|}=\frac{\alpha}{|1-\alpha|}
\end{array}\right\}
$$

A review of Eq. (10) to Eq. (14) indicates a complete definition of the similarity-based, relative-velocity model. To implement the numerical solutions, values of $\alpha$ were parametrically assigned from 0 (stationary fluid, moving surface) to $\infty$ (stationary surface, moving fluid).

\section{Numerical MethodS}

\subsection{Shooting Method Formulation}

With the definition of the state space variables, Eq. (11) and Eq. (12) along with their boundary conditions were converted to the following system of first order ordinary differential equations

$$
\begin{aligned}
& y_{1}=f, y_{2}=\frac{d f}{d \eta}, y_{3}=\frac{d^{2} f}{d \eta^{2}}, y_{4}=\theta, y_{5}=\frac{d \theta}{d \eta} \\
& y_{1}^{\prime}=y_{2} \quad y_{1}(0)=1 \\
& y_{2}^{\prime}=y_{3} \\
& y_{2}(0)=|1 /(1-\alpha)| \text {, } \\
& y_{3}^{\prime}=-0.5 y_{1} y_{3} \\
& y_{2}(\infty)=|\alpha /(1-\alpha)| \\
& y_{4}^{\prime}=y_{5} \\
& y_{3}(0)=\text { unknown } \\
& y_{5}^{\prime}=-0.5 \operatorname{Pr}\left(y_{1} y_{5}+y_{2} y_{4}\right) \\
& y_{4}(0)=1, y_{4}(\infty)=0 \\
& y_{5}(0)=\text { unknown }
\end{aligned}
$$

Two codes in Fortran 95 instructions were developed one with a fixed step size and the other with a variable step size.

\subsection{For Combined Shooting and Singular Perturbation Formulation}

For larger values of Prandtl number the energy equation becomes much stiffer or singularly perturbed and it is expected that the standard numerical methods fail to handle this situation unless special routines like MATLAB ode15s for stiff differential equations was used.

For convenience the formulation developed early by Habib and El-Zahar (2008) and El-Zahar and ELKabeir (2013) for singularly perturbed problems was applied for the energy equation as follows.

Let $\varepsilon=1 / \operatorname{Pr}$, Eq. (12) becomes

$\varepsilon \frac{d^{2} \theta}{d \eta^{2}}+\frac{1}{2} f \frac{d \theta}{d \eta}-\frac{d f}{d \eta} \theta=0$

Setting $\varepsilon=0$ one obtains the reduced problem

$\frac{1}{2} f \frac{d \bar{\theta}}{d \eta}=\frac{d f}{d \eta} \bar{\theta}, \quad \bar{\theta}(\infty)=0$

An asymptotic equivalent initial-value problem, to the boundary value problem, Eq. (17), is

$\varepsilon \frac{d \theta}{d \eta}+\frac{1}{2} f \theta=0, \quad \theta(0)=1$

Equation 19 was solved with the supply of the information obtained earlier for $f$ from the shooting methods.

\section{RESUlts AND Discussion}

Table 1 below shows the non-dimensional shear stress at the interface between the moving surface and the adjacent fluid at different values of $\alpha$ Inspection of Table 1 reveals that the direction of the wall shear depends on whether $U_{\infty}>U_{s}$ or $U_{s}>U_{\infty}$. In the former case, the fluid drags the sheet and, in turn, in the sheet tends to retard the motion of the fluid. Consequently the shear imposed by the sheet on the flowing fluid acts in the negative $\mathrm{x}$ direction, which accounts for the minus signs appearing in Table 1. On the other hand, the case of the faster moving sheet yields a force which the sheet exerts on the fluid in the positive $x$ direction, with corresponding plus signs in Table 1.

Table 1 Listing of the non-dimensional shear stress $f^{\prime \prime}(0)$ at $\operatorname{Pr}=100$.

\begin{tabular}{|c|c|c|r|r|r|c|}
\hline$\alpha=\frac{U_{f}}{U_{r l}}$ & 0.0 & 0.4 & 0.7 & 1.2 & 3 & 10 \\
\hline$f^{\prime \prime}(0)$ & -0.7864 & -0.9818 & -1.3066 & 1.6429 & 0.8892 & 0.7693 \\
\hline
\end{tabular}

Figure 2 shows a comparison of the non-dimensional shear stress, $f^{\prime \prime}(\eta)$ at different values of the relative velocity, $\alpha$. As observed from the figure, after certain value of $\alpha$ there is no effect on the shear stress. It 
was also found that the magnitude of $f^{\prime \prime}(\eta)$ was higher for the moving plate than for the stationary plate. This means that the shear stress, at the plate surface, being proportional to the magnitude of $f^{\prime \prime}(\eta)$ is higher for the moving plate. Figure 3 shows a comparison of the non-dimensional velocity profiles at different values of $\alpha$.

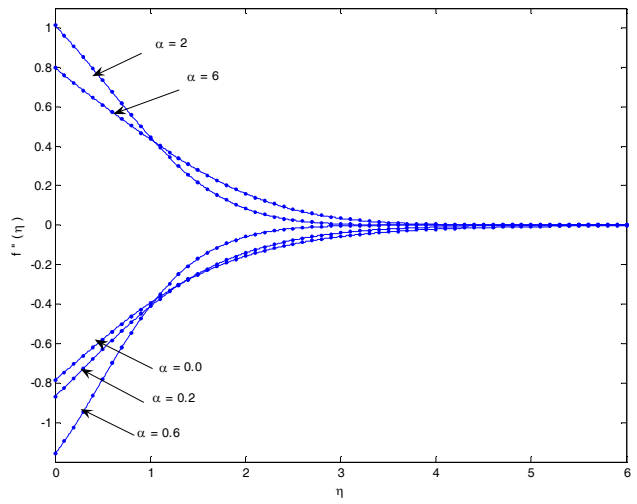

Fig. 2. A comparison of the non-dimensional shear stress, $f^{\prime \prime}(\eta)$ at $\operatorname{Pr}=100$ and different values of $\alpha$

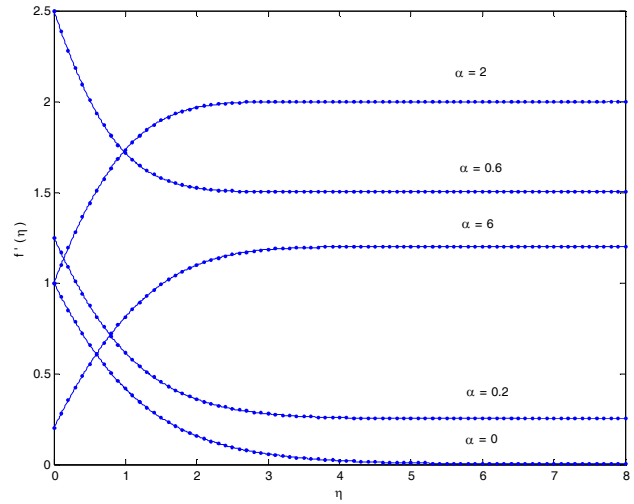

Fig. 3. A comparison of the velocity distribution across the boundary layer, $f^{\prime}(\eta)$ at $\operatorname{Pr}=100$ and different values of $\alpha$

The design of a thermal processing station for moving sheets requires knowledge of heat transfer rates and corresponding sheet temperature variations. This information is necessary for fixing the streamwise length of the processing station. Figure 4 shows the non-dimensional temperature profiles across the boundary at different values of $\alpha$ and for the case with no blowing using shooting method. It is observed that the magnitude of $\theta^{\prime}[0]$ is higher for the flow over a moving plate as compared to flow over a stationary plate. Therefore, the local heat transfer coefficient is higher for the moving surface. This means that, for a given fluid temperature $\theta$ and velocity $U_{s}$, the moving plate loses more energy to the flow than the semi-infinite stationary plate, with flow over it. The above result may be explained in terms of the transverse velocity $v$. Since this velocity $v$ is negative (i.e., toward the plate), the fluid at ambient temperature is brought toward the plate in the case of a continuously moving plate. This increases the temperature gradient at the surface and, hence, the heat transfer enhances.

Figure 5 shows a comparison of the solutions of the energy equation obtained by the shooting method to the corresponding results obtained from singularperturbation method. As illustrated in Fig. 5 the thermal layer becomes thinner compared to the previous case and temperature profiles are backed together. This is due to the blowing at the wall, which opposes the motion of the fluid toward the wall.

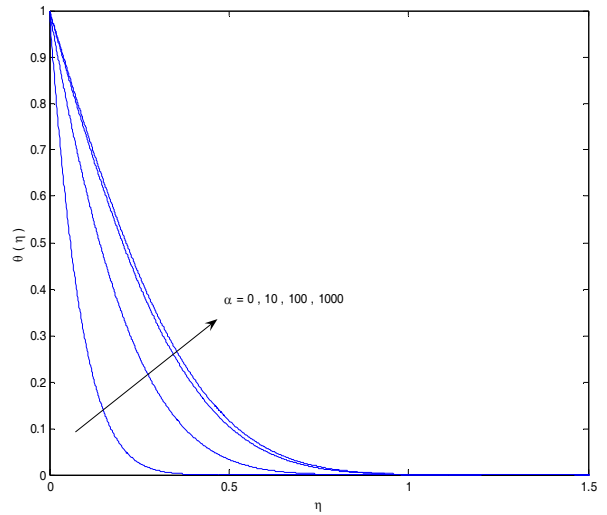

Fig. 4. Non-dimensional temperature profile, $\theta(\eta)$ across the boundary layer at $\operatorname{Pr}=100$ and different values of $\alpha$

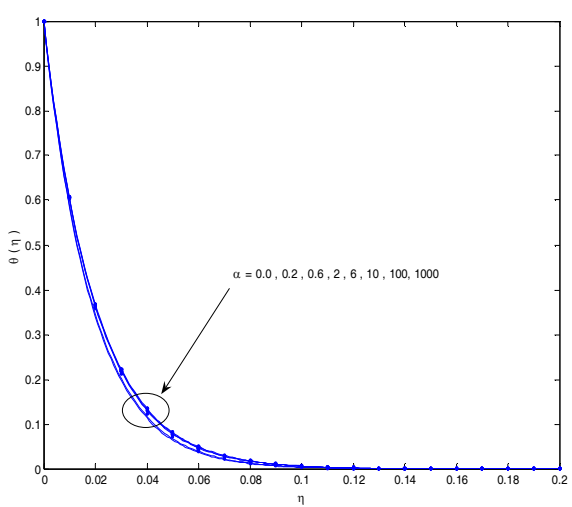

Fig. 5. A comparison of the Non-dimensional temperature profile, $\theta(\eta)$ across the boundary layer

at $\operatorname{Pr}=100$ and at different values of $\alpha$

\section{CONCLUSION}

An innovative procedure combining shooting and singular perturbation technique was used to solve the governing equations for the moving sheet in a moving fluid. For high values of Prandtl number, the energy equation became much stiffer or singularly perturbed and the standard numerical methods failed to handle it. A procedure for shooting based on Matlab ode15s for stiff differential equations was used for comparison purpose. The results show that the heat transfer depends on the relative velocity between the moving fluid and the moving sheet. If blowing is present the thermal 
H. M. Habib and E. R. El-Zahar / JAFM, Vol. 6, No. 3, pp. 369-373, 2013.

layer becomes thinner and temperature profiles are backed together.

\section{REFERENCES}

Abdelhafez, T.A. (1985). Skin friction and heat transfer on a continuous flat surface moving in a parallel free stream. International Journal of Heat Mass Transfer, 28, 1234-1237.

Abraham, J. P. and E. M. Sparrow (2005). Friction drag resulting from the simultaneous imposed motions of a free-stream and its bounding surface. International Journal of Heat Fluid Flow, 26(2), 289-295.

Abraham, J. P. and E. M. Sparrow (2005). Universal solutions for the stream-wise variation of the temperature of a moving sheet in the presence of a moving fluid. International Journal of Heat and Mass Transfer, 48, 3047-3056.

Char, M. I., C.O.K. Chen and J. W. Cleaver (1990). Conjugate forced convection heat transfer from a continuous moving flat sheet. International Journal of Heat Fluid Flow, 11, 257-261.

Chen, C.H. (1999). Forced convection over a continuous sheet with suction or injection moving in a flowing fluid. Acta Mechanica,. 138, 1-11.

Chen, C.H. (2000). Heat transfer characteristics of a non-isothermal surface moving parallel to a free stream. Acta Mechanica., 142(1-4), 195-205.

Chida, K. and Y. Katto (1976). Conjugate heat transfer of continuously moving surfaces. International Journal of Heat Mass Transfer, 19, 461-470.
Habib, H. M., and E. R. El-Zahar (2008). Variable step size initial value algorithm for singular perturbation problems using locally exact Integration. Applied Mathematics and Computation, 200(1), 330-340.

El-Zahar, E. R. and S. M. M. EL-Kabeir (2013). A new method for solving singularly perturbed boundary value problems. Applied Mathematics and Information Sciences, 7(3), 927-938.

Karwe, M.V. and Y. Jaluria, Y. (1992). Experimental investigation of thermal transport from a heated moving plate. International Journal of Heat Mass Transfer, 35, 493-511.

Karwe, M.V. and Y. Jaluria (1991, August). Numerical simulation of thermal transport associated with a continuously moving flat sheet in materials processing. Journal of Heat Transfer, 113(3), 612-619.

Karwe, M.V. and Y. Jaluria (1986). Thermal transport from a heated moving surface. Journal of Heat Transfer. 108(4), 728-733.

Mendez, F. and C. Trevino (2002). Heat transfer analysis on a moving flat sheet emerging into quiescent fluid. Journal of Thermophysics and Heat Transfer, 16(3), 373-378.

Sakiadis, B.C. (1961, March). Boundary layer behavior on continuous solid surfaces: I. Boundary layer equations for two dimensional and axisymmetric flow. AIChE Journal, 7(1), 26-28. 